\title{
Rosai-Dorfman disease as a rare cause of cervical lymphadenopathy - case report and literature review
}

\author{
ALEKSANDRA MIĘKUS ${ }^{1,2}$, JOANNA STEFANOWICZ ${ }^{1,2}$, GRAŻYNA KOBIERSKA-GULIDA ${ }^{3}$, \\ ELŻBIETA ADAMKIEWICZ-DROŻYŃSKA ${ }^{1,2}$
}

${ }^{1}$ Department of Paediatrics, Haematology, and Oncology, University Clinical Centre in Gdansk, Poland

${ }^{2}$ Department of Paediatrics, Haematology, and Oncology, Medical University of Gdansk, Poland

${ }^{3}$ Department of Pathomorphology, Medical University of Gdansk, Poland

\begin{abstract}
Rosai-Dorfman disease (RDD), also known as sinus histiocytosis with massive lymphadenopathy, is a rare, benign clinical entity of unknown cause. $R D D$ is characterised by the overproduction and accumulation of histiocytes, primarily in the lymph nodes, although it may affect every organ and system. It predominantly affects children and young adults. Typically, patients are in good general condition, with massive cervical lymphadenopathy and fever. In about $40 \%$ of cases extranodal localisation of RDD is diagnosed. In laboratory tests the most common abnormalities are increased erythrocyte sedimentation rate (ESR), leukocytosis with neutrophilia, normocytic anaemia, and hypergammaglobulinaemia. Histopathological examination remains the mainstay of diagnosis - lymph nodes have massive sinusoidal dilation, containing histiocytes positive for S-100 and CD68, and negative for CDIa. Most patients do not require treatment as spontaneous remissions are observed. We present a brief review of the literature and the case of a six-year-old boy with cervical lymphadenopathy diagnosed with RDD. So far, the patient has not required systemic treatment and has been kept under observation.
\end{abstract}

Key words: lymphadenopathy, Rosai-Dorfman disease, sinus histiocytosis.

(Centr Eur J Immunol 2018; 43 (3): 341-345)

\section{Introduction}

Rosai-Dorfman disease (RDD), also known as sinus histiocytosis with massive lymphadenopathy (SHML), is a rare, benign clinical entity, with symptoms that may be nonspecific. However, histopathology usually provides the right diagnosis.

In 1969 and 1972 Juan Rosai and Ronald F. Dorfman described a total of 34 cases of patients with painless, bilateral, massive cervical lymphadenopathy, fever, and distinctive abnormalities in laboratory tests: normochromic anaemia, leukocytosis with neutrophilia, increased erythrocyte sedimentation rate (ESR), and often hypergammaglobulinaemia. Subsequently, it was established as being a distinct clinical entity $[1,2]$.

RDD is characterised by the overproduction and accumulation of histiocytes, primarily in the lymph nodes, although it may affect every organ and system [3]. Extranodal localisation - usually occurring in the skin, orbit, central nervous system (CNS), and digestive system - is present in approximately $40 \%$ of cases. The peak incidence is in the second or third decade, and a male predominance is present. The aetiology of RDD is still unknown; possible caus- es include infection and/or immunological disorders [2]. Grossly, the excised lymph node is usually very large and is often matted to overlying soft tissues or adjacent lymph nodes. At low magnification, one sees a thickened as the source of matting. The lymph node architecture is dominated by a striking sinus expansion, filled with characteristic histiocyte-like cells. The cytoplasm of these cells often contains phagocytosed intact small lymphocytes (lymphophagocytosis) or a variety of cells, including red blood cells, neutrophils, or plasma cells (emperipolesis). Typically, there are multiple ingested cells forming a partial or complete ring around the nuclei. Late lesions may show extensive fibrosis. Immunohistochemical studies show that the proliferating cells express the histiocytic markers CD163 and CD68 as well as S-100 protein. They are negative for the Langerhans cell marker CD1a as well as the dendritic markers CD21 and CD35. They lack almost all lymphoid markers, although they may express CD30 in up to $50 \%$ of cases and show a germline configuration of their antigen receptor genes. They lack Birbeck granules on ultrastructural examination.

Correspondence: Joanna Stefanowicz, MD, PhD, Department of Paediatrics, Haematology, and Oncology, Medical University of Gdansk,

7 Dębinki St., 80-850 Gdansk, Poland, e-mail: jstefanowicz@ gumed.edu.pl

Submitted: 23.08.2017; Accepted: 22.11.2017 
There are no unified therapeutic algorithms for RDD. Due to the fact that in many cases spontaneous regression is observed, usually the "watch and wait" approach is used, meaning that surgical and systemic (e.g. steroids, chemotherapy) treatment is rarely required [4].

This article will present the case of a six-year-old patient diagnosed with RDD and a brief review of the literature.

\section{Case report}

A six-year-old boy was admitted to the Children's Oncology Department in Gdansk for diagnostics because of cervical lymphadenopathy. In the medical history lymphadenopathy had been observed on the left side for four months. The patient had good general condition without fever or other alarming symptoms. From the age of three years he had been under observation at a Pulmonology Clinic because of a constant, hacking cough. Under physical examination the only abnormality observed was significant cervical asymmetry caused by a pathological mass on the left side (Fig. 1). The mass was firm, painless, $50 \times 40 \mathrm{~mm}$ in size, and the skin in the area was without changes. On the right side, small lymph nodes were palpable. Laboratory tests showed slightly elevated C-reactive protein (CRP) $(8.56 \mathrm{mg} / \mathrm{l})$ and fibrinogen $(3.8 \mathrm{G} / \mathrm{l})$ levels, increased ESR $(49 \mathrm{~mm} / \mathrm{h})$, decreased $\mathrm{Hgb}(10.6 \mathrm{~g} / \mathrm{dl})$ and iron (24 ug/dl) levels, Ht 30.7\%, and mean corpuscular volume (MCV) and ferritin at the lower limit. Anti-CMV antibodies were $\operatorname{IgG}(+)$ and $\operatorname{IgM}(-)$. EBV and toxoplasmosis antibodies were negative, with HIV also negative. The ANA-Hep-2 test was positive (1: 640, SSA/SSB); rheumatoid factor (RF) and immunoglobulin levels were normal. Under ultrasound many pathological lymph nodes

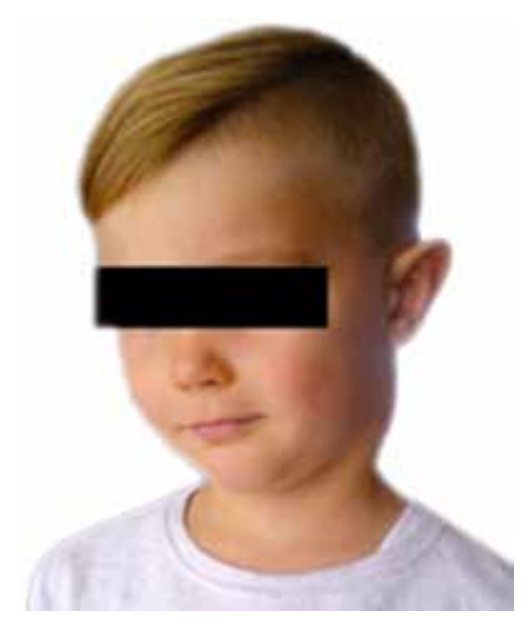

Fig. 1. Lymphadenopathy in patient with Rosai-Dorfman disease were observed, CT scan images suggested lymphadenopathy caused by systemic disease or mononucleosis. Bone scintigraphy showed increased uptake in left temporal bone and right frontal sinus. To verify those areas an MRI of the head was performed - inflammatory changes in the sinus and worse pneumatisation of temporal bones were described. Apart from the cervical lymph nodes, no other localisation of the disease was found. During hospitalisation the patient became infected with chickenpox.

Histopathology showed lymph nodes with an abnormal architecture, thickened capsule, and paracortical hyperplasia accompanied by vascular proliferation. The paracortex was infiltrated with pleomorphic lymphocytes, plasma cells, and neutrophils. Some of the germinal centres were hyperactive, others had features of atrophy. Sinuses in the lymph nodes were massively dilated, filled with histiocyte-like cells with abundant, foamy cytoplasm. These cells had an intermediate to large-sized round nuclei, delicate nuclear membranes, and a vesicular chromatin pattern with one to a few moderately prominent nucleoli. Emperipolesis was observed. Plasma cells were abundant, both adjacent to the proliferating histiocytes within the sinuses as well as within the adjacent expanded medullary cords. Immunohistochemical stains showed that the proliferating cells were $\mathrm{S}-100^{+}, \mathrm{CD}^{+} 8^{+}, \mathrm{CD} 1 \mathrm{a}^{-}$, and parvovirus B-19 antigen positive. Rosai-Dorfman disease was diagnosed.

Due to his excellent general condition and stable lymph node image under ultrasound examination, we decided to keep the patient under observation.

\section{Literature review}

\section{Epidemiology and aetiology}

RDD is a rare disorder, and since 1969 fewer than 1000 cases have been reported in medical literature [3]. It can affect individuals of any age, but the peak incidence is in the second or third decade. RDD is more common in males and people of African descent $[2,4,5]$.

The aetiology of RDD is unknown. Over the years there have been many theories attempting to explain RDD's pathogenesis, but each of them has led to conflicting results. Researchers have indicated infectious agents as one of the potential causes of RDD, for instance HHV-6 [6, 7], HHV-8, parvovirus B19, EBV [7], CMV, VZV, Brucella, and Klebsiella; however, there is no conclusive evidence to confirm this theory. In some cases, the HHV-6 antigen has been identified in the pathological histiocytes, while EBV and parvovirus B19 have been present in lymphocytes, which may eventually be phagocytosed by histiocytes [4]. In one of the articles suggested by the authors, it was observed that primarily infected lymphocytes induce a secondary histiocytic reaction that eventually causes the characteristic microscopic image of RDD [8]. 
Table 1. Recommended diagnostic tests in patients with Rosai-Dorfman disease

\begin{tabular}{|c|c|c|}
\hline Laboratory tests & Virology & Imaging tests \\
\hline $\begin{array}{l}\text { Full blood count } \\
\text { CRP, ESR } \\
\text { Renal panel } \\
\text { Liver panel } \\
\text { D-dimer, coagulogram } \\
\text { Fibrinogen } \\
\text { Ferritin } \\
\text { Proteinogram } \\
\text { Immunoglobulin levels } \\
\text { RF, ANA } \\
\text { In patients with abnormalities in } \\
\text { peripheral blood smear - bone marrow } \\
\text { aspiration }\end{array}$ & $\begin{array}{c}\text { HHV-6, HHV-8 } \\
\text { HIV } \\
\text { EBV } \\
\text { CMV } \\
\text { Parvovirus b-19 } \\
\text { VZV }\end{array}$ & $\begin{array}{l}\text { USG of suspicious lymph nodes, mediastinum, abdomen } \\
\text { CT of head, neck, chest, abdomen, pelvis with IV contrast } \\
\text { Bone scintigraphy } \\
\text { In patients with neurological symptoms - MRI of the head with } \\
\text { IV contrast } \\
\text { PET-FDG - rarely; may be helpful to check early response in the } \\
\text { treatment [5] }\end{array}$ \\
\hline
\end{tabular}

Other potential causes may be broadly-defined immunological disorders (e.g. immunodeficiency, autoimmune diseases). In view of the fact that some patients have hypergammaglobulinaemia, scientists have been looking for a relationship between RDD and IgG4-related diseases, but detailed analyses have put this theory into question $[4,9,10]$.

One of the more recent studies considers the coexistence of Langerhans cell histiocytosis ( $\mathrm{LCH}$ ) and RDD [11-13]. Although under WHO classification LCH and RDD are separate, it has been emphasised that both diseases share the same bone marrow precursor cell, which later undergoes further differentiation [12]. Common features of histiocytes in these two disorders are, i.a., positive reaction to $\mathrm{S}-100$ staining, and expression of cathepsin D and $\mathrm{E}$ [12]. There are reports of patients treated for $\mathrm{LCH}$, in whom after chemotherapy RDD is revealed. This may be caused by immunological abnormalities connected with one disease, eventually leading to the disclosure of another one, or, alternatively, exposure to systemic chemotherapy causes disturbed differentiation in cells. In one of the reported cases, the authors observed histiocytes with immunohistochemical phenotype typical for $\mathrm{LCH}\left(\mathrm{S}_{100}\right.$, $\mathrm{CD} 1 \mathrm{a}^{+}$, presence of Birbeck granules), which underwent changes during chemotherapy $\left(\mathrm{S}_{100}+\mathrm{CD}^{+} \mathrm{a}^{+}\right.$, no Birbeck granules), finally presenting a profile typical for RDD $\left(\mathrm{S} 100^{+}, \mathrm{CD} 1 \mathrm{a}^{-}\right)[12,14]$. There are also patients diagnosed with RDD during or after treatment for leukaemia, Hodgkin lymphoma, and non-Hodgkin lymphoma [5, 15, 16].

Scientists are also looking for genetic disorders that could be responsible for RDD development. The literature refers to, i.a., SLC29A3 mutations and familial RDD [4, 17, 18].

\section{Symptoms}

Due to the fact that RDD may affect every organ and system, symptoms vary depending on the localisation of the disease. The most frequent clinical presentation of RDD is massive, bilateral, painless cervical lymphadenopathy accompanied by fever [2]. In most cases the patients are in good general condition, although night sweats and weight loss may also be observed. As a result, RDD can sometimes mimic lymphoma [4].

Extranodal disease is diagnosed in about $40 \%$ of cases [4]. Some patients may have extranodal RDD without the presence of lymphadenopathy [3]. The most common extranodal site is the skin (painless, follicular lesions, mainly on the head and neck) [19], orbit [20], CNS (predominantly dural-based intracranial mass) [17, 21, 22], digestive system [6], and salivary glands [23]. Hepatosplenomegaly is uncommon. Manifestation of RDD in the heart is very rare. It can reveal as a pericardial mass or a mass in the heart, which could be easily mistaken for a myxoma on clinical and radiological evaluation [24, 25].

\section{Diagnostics and results of laboratory tests}

Patients with RDD often have distinctive abnormalities present in laboratory tests. Increased ESR is observed in about $90 \%$ of cases. In a complete blood count, the most common anomalies are leukocytosis with neutrophilia, a mild normochromic normocytic anaemia, and rarely haemolytic anaemia and eosinophilia [4]. The ferritin level is usually normal or slightly elevated. Increased IgG and RF levels, and positive antinuclear antibody (ANA) tests are frequent [4]. Staging should include additional laboratory and imaging tests (Table 1) [5].

Medical history and physical examination remain very important steps in diagnosing RDD, but because of its rarity and heterogeneous symptoms, usually only microscopic examination gives a correct diagnosis.

\section{Histopathology}

Under microscopic examination lymph nodes have a thickened capsule, abnormal architecture, and massive sinus expansion. The sinuses are filled with the characteristic proliferating histiocyte-like cells. The hallmark of RDD is lymphophagocytosis or emperipolesis - the phagocytosis of a variety of intact cells, including red blood cells, neu- 
trophils, or plasma cells [11]. Immunohistochemical stains are crucial in confirming a diagnosis. The typical RDD phenotypes are: $\mathrm{S}_{100^{+}}, \mathrm{CD}^{+} 8^{+}, \mathrm{CD}_{163}{ }^{+}, \alpha-1$-antitripsin ${ }^{+}$, $\alpha$-1-antichymotripsin ${ }^{+}$, and $\mathrm{CD}_{1 \mathrm{a}}{ }^{-}[4,11]$. BRAF V600E mutation and Birbeck granules, both found in $\mathrm{LCH}$, are negative in RDD [4].

\section{Course of illness and prognosis}

The course of the illness is unpredictable. Classic symptoms, such as cervical lymphadenopathy, fever, and good general condition, may persist from several weeks to even a few years (the average time is 3-9 months) [2]. Without treatment, relapsing-remitting RDD will occur in $70 \%$ of cases, spontaneous regression in $20 \%$, and in $10 \%$ there will be a progression of the disease [4]. It is recommended that a clinical examination and laboratory tests be performed every 3-6 months during the first two years following diagnosis, then every year [4].

In 2011 the Histiocyte Society divided RDD patients into three major groups: 1) Patients with sudden enlargement of the lymph nodes, in which spontaneous regression is observed, without any further recurrences - consistent with the best prognosis; 2) Patients with immunological abnormalities, in which lymphadenopathy is more generalised - the prognosis being worse; and 3) Patients with extranodal site involvement and/or multinodal disease, with repeated relapses and remissions over a period of years - the prognosis depends on the type and number of extranodal sites [5].

Cutaneous RDD (CRDD) was established as a separated clinical entity, in which only skin is involved - patients do not present lymphadenopathy. CRDD usually affects individuals around the age of 50 years, predominantly women, especially from the Caucasian population, and is associated with an excellent prognosis $[3,4,19]$.

RDD in most cases is a benign disorder; however, multiorgan involvement or dysfunction and association with immune dysfunction are poor prognostic indicators and may lead to death. The most common causes of reported deaths were identified immunological abnormalities, severe infections, surgical complications, complications after radiotherapy, and the compression of airways by enlarged lymph nodes $[2,26]$. There are reports of RDD leading to lymphoma, amyloidosis, and consequently death resulting from these diseases [26].

\section{Treatment}

Because of its rarity, there are no unified therapeutic algorithms for RDD. Spontaneous remission is often observed; therefore, the "watch and wait" approach is recommended [3, 4]. The majority of patients do not require treatment, although it should be considered in cases where the disease affects vital organs/systems or lymph node masses obstruct the airways/spinal cord [4, 5, 27].
The main method of treatment in RDD is surgery. Due to the small number of patients given systemic treatment or radiotherapy, the effectiveness of these methods in RDD remains uncertain. For patients requiring systemic treatment, the established first-line therapeutic option (both in nodal and extranodal localisation of RDD) is steroids [3-5, 11, 28]. However, there are no standard guidelines regarding the duration for which they should be used and in what dose [4].

Chemotherapy was administered to patients with disseminated disease, who had not responded to other therapeutic methods. Numerous chemotherapeutic agents were used $[4,5,11]$, i.e. vinca alkaloids, alkylating agents, anthracyclines, cladribine [29], clofarabine [30], methotrexate [31], mercaptopurine [31], azathioprine [32], and chlorodeoxyadenosine [33]. There are also reports of treatment with interferon $\alpha$ [34], rituximab [35, 36], imatinib [37], and retinoids [38].

Radiotherapy was considered a palliative method in patients with symptomatic RDD [4], but according to the latest findings it can give better results than chemotherapy in some cases [5, 11]. Radiotherapy appears to be an alternative in steroid-resistant patients [39, 40].

There are reports describing RDD patients with a high level of HHV-6/VZV antibody titres, in which there was significant improvement after the application of acyclovir $[5,6,41]$. In other cases, complete remission was observed after using thalidomide [5, 11].

\section{The authors declare no conflict of interest.}

\section{References}

1. Rosai J, Dorfman RF (1969): Sinus histiocytosis with massive lymphadenopathy: A newly recognized benign clinicopathologic entity. Arch Pathol 87: 63-70.

2. Rosai J, Dorfman RF (1972): Sinus histiocytosis with massive lymphadenopathy: a pseudolymphomatous benign disorder. Analysis of 34 cases. Cancer 30: 1174-1188.

3. National Organization for Rare Disorders. Rosai-Dorfman Disease http://rarediseases.org/rare-diseases/rosai-dorfman-disease/ (accessed: February 10, 2016).

4. Dalia S, Sagatys E, Sokol L, Kubal T (2014): Rosai-Dorfman Disease. Tumor Biology, Clinical Features, Pathology and Treatment. Cancer Control 21: 322-327.

5. Histiocyte Society 2011; Rosai-Dorfman Disease Rewiev: Outline. https://www.histiocytesociety.org/document. doc?id=54 (accessed: February 10, 2016).

6. Lauwers GY, Perez-Atayde A, Dorfman RF, Rosai J (2000): The Digestive System Manifestations of Rosai-Dorfman Disease (Sinus Histiocytosis With Massive Lymphadenopahy): Review of 11 Cases. Hum Pathol 31: 380-385.

7. Levine PH, Jahan N, Murari P, et al. (1992): Detection of human herpesvirus 6 in tissues involved by sinus histiocytosis with massive lymphadenopathy (Rosai-Dorfman disease). JID 166: 291-295. 
8. Mehraein Y, Wagner M, Remberger K, et al. (2006): Parvovirus B19 detected in Rosai-Dorfman disease in nodal and extranodal manifestations. J Clin Pathol 59: 1320-1326.

9. Tseng-Tong K, Tse-Ching C, Li-Yu L, Pei-Hsuan L (2009): IgG4-positive plasma cells in cutaneous Rosai-Dorfman disease: an additional immunohistochemical feature and possible relationship to IgG4-related sclerosing disease. J Cutan Pathol 36: 1069-1073.

10. Liping L, Perry AM, Wenfeng C, et al. (2013): Relationship Between Rosai-Dorfman Disease and IgG4-Related Disease. Study of 32 cases. Am J Clin Pathol 140: 395-402.

11. Maia RC, de Meis E, Romano S, et al. (2015): Rosai-Dorfman Disease: a report of eight cases in a tertiary care center and a review of the literature. Braz J Med and Biol Res 48: 6-12.

12. Cohen-Barak E, Rozenman D, Schafer J, et al. (2014): An unusual co-occurrence of Langerhans cell histiocytosis and Rosai-Dorfman disease: report of a case and rewiev of the literature. Int J Dermatol 53: 558-563.

13. Wang KH, Cheng CJ, Hu CH, Lee WR (2002): Coexistence of localized Langerhans cell histiocytosis and cutaneous Rosai - Dorfman disease. Br J Dermatol 147: 770-774.

14. Patrizi A, Neri I, Bianchi F (2004): Langerhans cell histiocytosis and juvenile xanthogranuloma. Two case reports. Dermatology 209: 57-61.

15. Moore JC, Zhao X, Nelson EL (2008): Concomitant sinus histiocytosis with massive lymphadenopathy (Rosai-Dorfman Disease) and diffuse large B-cell lymphoma: a case report. J Med Case Reports 2: 1-7.

16. Lu D, Estalilla OC, Manning Jr JT, Medeiros J (2000): Sinus Histiocytosis with Massive Lymphadenopathy and Malignant Lymphoma Involving the Same Lymph Node: A Report of Four Cases and Review of the Literature. Mod Pathol 13: 414-419.

17. Lewis Cooper S, Jenrette JM (2012): Rosai-Dorfman Disease: Management of CNS and Systemic Involvement. CAH\&O 10: 199-202.

18. Morgan NV, Morris MR, Cangul H, et al. (2010): Mutations in SLC29A3, Encoding, and Equilibrative Nucleoside Transporter ENT3, Cause a Familial Histiocytosis Syndrome (Faisalabad Histiocytosis) and Familial Rosai-Dorfman Disease. PLoS Genetics 6: 1-9.

19. Brenn T, Calonje E, Granter S, et al. (2002): Cutaneous Rosai-Dorfman Disease Is a Distinct Clinical Entity. Am J Dermatopathol 24: 385-391.

20. Vemuganti GK, Naik MN, Honavar SG (2008): Rosai Dorfman disease of the orbit. J Hematol Oncol 28: 1-7.

21. Sandoval-Sus JD, Sandoval-Leon AC, Chapman JR, et al. (2014): Rosai-Dorfman Disease od the Central Nervous System. Report of 6 Cases and Review of the Literature. Medicine 93: 165-175.

22. Warrier R, Cahuhan A, Jewan Y, et al. (2012): Rosai-Dorfman Disease With Central Nervous System Involvement. CAH\&O 10: 196-198.

23. Guven G, Ilgan S, Altun C, et al. (2007): Rosai - Dorfman disease of the parotid and submandibular glands:salivary gland scintigraphy and oral findings in two siblings. DMFR 36: 428-433.

24. Lao IW, Dong Y, Wang J (2014): Rosai-Dorfman disease of the pericardium: a case report and review of literature. Int J Clin Exp Pathol 7: 3408-3412.

25. Heidarian A, Anwar A, Haseeb MA, Gupta R (2017): Extranodal Rosai-Dorfman disease arising in the heart: clinical course and review of literature. Cardiovasc Pathol 4: 31:1-4.
26. Foucar E, Rosai J, Dorfman RF (1984): Sinus Histiocytosis With Massive Lymphadenopathy. An Analysis of 14 Deaths Occuring in a Patient Registry. Cancer 54: 1834-1840.

27. Pulsoni A, Anghel G, Falcucci P, et al (2002): Treatment of Sinus Histiocytosis With Massive Lymphadenopathy (Rosai-Dorfman Disease): Report of a Case and Literature Rewiev. Am J Hematol 69: 67-71.

28. McPherson CM, Brown J, Kim AW, DeMonte F (2006): Regression of intracranial Rosai-Dorfman disease following corticosteroid therapy. J Neurosurg 104: 840-844.

29. Aouba A, Terrier B, Vasiliu V, et al. (2006): Dramatic clinical efficacy of cladribine in Rosai-Dorfman disease and evolution of the cytokine profile: a new therapeutic approach. Haematologica 91: 144-145.

30. Simko SJ, Tran HD, Jones J, et al. (2014): Clofarabine salvage therapy in refractory multifocal histicytic disorders, incluging Langerhans cell histiocytosis, juvenile xanthogranuloma and Rosai-Dorfman disease. Pediatr Blood Cancer 61: 479-487.

31. Horneff G, Jurgens H, Hort W, et al. (1996): Sinus histiocytosis With Massive Lymphadenopathy (Rosai-Dorfman Disease): Response to Methotrexate and Mercaptopurine. Med Pediatr Oncol 27: 187-192.

32. Le Guenno G, Galicier L, Uro-Coste E, et al. (2012): Successful treatment with azathioprine of relapsing Rosai-Dorfman disease of the central nervous system. J Neurosurg 117: 486-489.

33. Konca C, Ozkurt ZN, Deger M, et al. (2008): Extranodal multifocal Rosai-Dorfman disease: response to 2-chlorodeoksyadenosine treatment. Int J Hematol 89: 58-62.

34. Lohr HF, Godderz W, Wolfe T, et al. (1995): Long-term Survival in a Patient with Rosai-Dorfman Disease Treated with Interferon-alfa. Eur J Cancer 31: 2427-2428.

35. Alqanatish JT, Houghton K, Bond M, et al. (2016): Rituximab Treatment in a Child with Rosai-Dorfman Disease and Systemic Lupus Erythematosus. J Rheumatol 37: 1783-1784.

36. Pagel JM, Lionberger J, Gopal AK, et al. (2007): Therapeutic use of Rituximab for sinus histiocytosis with massive lymphadenopathy (Rosai-Dorfman disease). Am J Hematol 82: 1121-1122.

37. Gebhardt C, Averbeck M, Paasch U, et al. (2009): A case of cutaneous Rosai-Dorfman disease refractory to imatinib therapy. Arch Dermatol 145: 571-574.

38. Mebazaa A, Trabelsi S, Denguezli M, et al. (2007): Extensive purely cutaneous Rosai-Dorfman disease responsive to acitretin. Int J Dermatol 46: 1208-1210.

39. Maklad AM, Bayoumi Y, Tunio M, et al. (2013): Steroid-Resistant Extranodal Rosai-Dorfman Disease of Cheek Mass and Ptosis Treated with Radiation Therapy. Case Rep Hematol ID: 428297.

40. Toguri D, Louie AV, Rizkalla K, et al. (2011): Radiotherapy for steroid-resistant laryngeal Rosai-Dorfman disease. Curr Oncol 18: 158-162.

41. Baildam EM, Ewing CI, D’Souza SW, Stevens RF (1992): Sinus histiocytosis with massive lymphadenopathy (Rosai-Dorfman disease): response to acyclovir. J R Soc Med 85: 179-180. 